\title{
Thermography in High-Intensity Focused Ultrasound Ablation
} Yufeng Zhou

School of Mechanical \& Aerospace Engineering, Nanyang Technological University, Singapore

Application of high-intensity focused ultrasound (HIFU) in therapeutic medicine has started in the 1950s for the treatment of Parkinson's disease. With the development of medical diagnosis, such as ultrasound imaging and magnetic resonance imaging (MRI), automatic control, imaging processing and electrical technology, extracorporeal and intrarectal HIFU systems have been developed for clinical trials since the middle of 1990s. Available targets include: prostate, pancreatic, liver, kidney, brain, bone, breast cancers and uterine fibroids. In Asia and Europe more than 100,000 patients have been involved with promising results. In comparison to conventional open surgery, radio- or chemo-therapy, HIFU has the advantages of non-invasiveness, non-ionization, few complications, easy recoveries and potentially infinite number of sessions $[1,2]$. HIFU was selected by TIME magazine as one of the 50 most inspired ideas, innovations and revolutions of 2011 [3]. The principle of HIFU is that the absorption of acoustic energy by the internal tissue leads to the temperature elevation over $65^{\circ} \mathrm{C}$ to denature the protein in the solid tumor/cancer for the generation of coagulative necrosis. The beam size of HIFU is usually 1-2 $\mathrm{mm}$ in the lateral and $1 \mathrm{~cm}$ in the axial directions, respectively. The boundary between apparently totally disrupted cells and normal tissue is no more than $50 \mu \mathrm{m}$ in width. No damage on the intervening tissue (i.e., artery, nerve and skin surface) is found in the clinics if acoustic coupling is kept good during the ablation. Since the major mechanism of HIFU is the thermal effect, accurate and real-time temperature monitoring at the HIFU focal region is of importance for the efficacy and efficiency in clinical HIFU application as well as the medical device approval by Food and Drug Administration (FDA) of United States.

There are two imaging modalities integrated with HIFU to detect the region-of-interest: ultrasound and MRI. Ultrasound imaging uses the same energy modality and pathway as therapeutic pulse for HIFU ablation guidance and lesion monitoring, so unsatisfactory acoustic coupling or blocking by ribs or other organs could be identified. Despite the low cost and easy integration of ultrasound diagnostic system, the image quality itself may not be sufficient to detect solid tumor/cancer without registration with other diagnosis information (i.e., CT, MRT and PET). In contrast, MRI has excellent anatomical resolution and high sensitivity for tumor detection, offering accurate planning of the tissue to be targeted, it's bulky, expensive and operation complicated. Thereby current temperature monitoring technique for HIFU ablation is based on either ultrasound or MRI.

Ultrasound based temperature imaging was proposed as a means to noninvasively measure the deposition of heat by tracking the echo shifts in the backscattered ultrasound signals from the target undergoing ablation, which are due to the combination of the local temperature dependence of sound speed and thermal expansion in the heated region [4]. There is a linear relationship between these shifts and the tissue temperature rise, but only in the low temperature rise $\left(\sim 10^{\circ} \mathrm{C}\right)$ for both two and three dimensional assessment [4-6]. Echo shifts are estimated from the correlation of successive backscattered ultrasound frames using a standard diagnostic scanner. The accuracy of $0.5^{\circ} \mathrm{C}$ and spatial resolution of $2 \mathrm{~mm}$ were evaluated experimentally in tissue mimicking phantoms and ex vivo tissues by comparison with measurements from thermocouples. In addition, a high-resolution spectral estimation method for tracking frequency shifts at two or more harmonic frequencies associated with temperature change was also available with comparable performance as the echo shift method [7]. Changes in backscattering energy CBE in motion-compensated images was nearly monotonic, with a maximum change of $4-5 \mathrm{~dB}$, as proved in both theoretical simulation [8] and measurement [9]. CBE depends on tissue inhomogeneity, which makes calibration difficult. Although ultrasound thermography technique demonstrates potential in imaging guidance of thermal therapy, determining the location of the therapeutic focal spot and assessing the extent of the heated region are only valid for subablative intensities.

During the HIFU ablation, noninvasive three-dimensional mapping of temperature changes is feasible with MRI based on the relaxation time $T_{1}$, the water molecular apparent diffusion coefficient $(A D C)$, or proton resonance frequency (PRF) of tissue water [10]. PRF appears as a preferred approach to measure temperature with MRI at mid to high magnetic field strength ( $\geq 1$ Tesla) because of the excellent linearity and near-independence with respect to tissue type, together with good temperature sensitivity. A standard deviation of less than $1^{\circ} \mathrm{C}$ for a temporal resolution below $1 \mathrm{~s}$ and a spatial resolution of about $2 \mathrm{~mm}$ is feasible for a single slice of immobile tissue. MRI thermography enables a proper control of the treatment progress using the thermal dose calculated from the temperature history, which ensures that healthy tissues around the tumor are spared and thermal dose reliably shows in real time how treatment progress and indicates when the therapy endpoint is reached. Because of volumetric acquisitions, excellent contrast and above all temperature imaging, MRI demonstrates major advantages in terms of monitoring HIFU ablation. However, MRI is extremely sensitive to radiofrequency interferences (hence the Faraday cage around it), magnetic susceptibility variations, motion artifacts and displacement registration between scans. Therefore, in order to profit from all the advantages of MRI, the HIFU system has to be specifically designed for active compatibility with the MRI environment. However, because of the relatively large spatial resolution and low frame rate of MRI thermography, MRI-determined temperature values may be underestimated. In a 4.7-Tesla magnet the temperature measured in a single MRI voxel by water PRF shift attained a maximum value of only $73^{\circ} \mathrm{C}$ after $7 \mathrm{~s}$ of continuous HIFU exposure when boiling in the gel phantom started using a 2-MHz HIFU source. Nonlinear modeling of the acoustic field combined with a heat-transfer equation predicted $100^{\circ} \mathrm{C}$ after $7 \mathrm{~s}$ of exposure. Averaging of the calculated temperature field over the volume of the MRI voxel $\left(0.3 \times 0.5 \times 2 \mathrm{~mm}^{3}\right)$ yielded a

${ }^{*}$ Corresponding author: Zhou Yufeng, Assistant Professor, School of Mechanical \& Aerospace Engineering, Nanyang Technological University, Singapore, E-mail: fzhou@ntu.edu.sg

Received August 09, 2011; Accepted December 12, 2011; Published December 14,2011

Citation: Zhou Y (2012) Thermography in High-Intensity Focused Ultrasound Ablation. J Appl Mech Eng 1:e101. doi:10.4172/2168-9873.1000e101

Copyright: (c) 2012 Zhou Y. This is an open-access article distributed under the terms of the Creative Commons Attribution License, which permits unrestricted use, distribution, and reproduction in any medium, provided the original author and source are credited. 
maximum of $73^{\circ} \mathrm{C}$ that agreed with the MRI thermometry measurement [11].

In summary, the thermography is an important feature of ensuring the efficacy and efficiency in HIFU ablation as well as wide acceptance of HIFU by both physicians and patients. The current temperature measurement methods by ultrasound signals have a satisfactory resolution in low temperature elevation at a low cost. However, due to the nonlinear effects of the tissue properties at high temperature, application of this method in HIFU ablation is of difficulty. In addition, the vulnerability to motion artifacts and tissue deformation limit the quantitative assessment. Therefore, one of the commercial HIFU systems only illustrates the low, medium and high degree of change in backscattered energy levels to provide qualitative information [12]. In contrast, although the cost of MRI is quite high, the ability of providing real-time thermography makes MRI the sole HIFU guidance modality approved by FDA. With the further understanding of thermometry of MRI in HIFU field, it is found that MRI-determined temperature values may be under-estimated because of the spatial-averaging effect. Therefore, technical improvement and development in thermography for HIFU ablation are in a great need to translate this innovative noninvasive cancer treatment modality into a wide clinical application.

\section{References}

1. Ter Haar GR (2001) Acoustic Surgery. Physics Today: 29-34.

2. Zhou YF, Kargl SG, Hwang JH (2011) The effect of the scanning pathway in high-intensity focused ultrasound therapy on lesion production. Ultrasound in Medicine and Biology 37: 1457-1468.

3. Brock-Abraham C, Carbone N, Dodds E, Kluger J, Park A, et al. (2011) The 50 Best Inventions. Time 178: 55-82.
4. Simon C, VanBaren P, Ebbini ES (1998) Two-dimensional temperature estimation using diagnostic ultrasound. IEEE Transactions on Ultrasonics, Ferroelectrics and Frequency Control 45: 1088-1099.

5. Anand A, Savery D, Hall C (2007) Three-dimensional spatial and tempora temperature imaging in gel phantoms using backscattered ultrasound. IEEE Transactions on Ultrasonics, Ferroelectrics and Frequency Control 54: 23-31.

6. Daniels MJ, Varghese T, Madsen EL, Zagzebski JA (2007) Non-invasive ultrasound-based temperature imaging for monitoring radiofrequency heatingphantom results. Physics in Medicine and Biology 52: 4827-4843.

7. Amini AN, Ebbini ES, Georgiou TT (2005) Noninvasive estimation of tissue temperature via high-resolution spectral analysis techniques. IEEE Transactions on Biomedical Engineering 52: 221-228.

8. Arthur RM, Straube WL, Starman JD, Moros EG (2003) Noninvasive temperature estimation based on the energy of backscattered ultrasound. Medical Physics 30: 1021-1029.

9. Arthur RM, Trobaugh J, Straube WL, Moros EG (2005) Temperature dependence of ultrasonic backscattered energy in motion compensated images. IEEE Transactions on Ultrasonics, Ferroelectrics and Frequency Control 52: 1644-1652

10. Bohris C, Schreiber WG, Jenne J, Simiantonakis I, Rastert R, et al. (1999) Quantitative MR temperature monitoring of high-intensity focused ultrasound therapy. Magnetic Resonance Imaging 17: 603-610.

11. Khokhlova TD, Canney MS, Lee D, Marro K, I, Crum LA, et al. (2009) Magnetic resonance imaging of boiling induced by high intensity focused ultrasound. $J$ Acoust Soc Am 125: 2420-2431.

12. Chen W-H, Sanghvi NT, Carlson RF, Uchida T (2010) Real-time tissue change monitoring on the Sonablate 500 during high-intensity focused ultrasound (HIFU) treatment of prostate cancer. 10th International Symposium on Therapeutic Ultrasound. Tokyo: AIP 391-396. 